\title{
Radial migration in galactic disks caused by resonance overlap of multiple patterns: Self-consistent simulations
}

\author{
I. Minchev ${ }^{1,2}$, B. Famaey ${ }^{2,3}$, F. Combes ${ }^{4}$, P. Di Matteo ${ }^{5}$, M. Mouhcine ${ }^{2,6}$, and H. Wozniak ${ }^{2}$ \\ 1 AIP, An der Sterwarte 16, 14482 Potsdam, Germany \\ 2 Observatoire Astronomique de Strasbourg, CNRS UMR 7550, 67000 Strasbourg, France \\ e-mail: ivan . minchev@astro. unistra.fr \\ 3 AIfA, Universtät Bonn, 53121 Bonn, Germany \\ 4 Observatoire de Paris, LERMA, 61 avenue de L'Observatoire, 75014 Paris, France \\ 5 Observatoire de Paris-Meudon, GEPI, CNRS UMR 8111, 5 pl. Jules Janssen, 92195 Meudon, France \\ 6 ARI, Liverpool John Moores University, Twelve Quays House, Egerton Wharf, Birkenhead, CH41 1LD, UK
}

Received 2 June 2010 / Accepted 7 December 2010

ABSTRACT

\begin{abstract}
We have recently identified a new radial migration mechanism resulting from the overlap of spiral and bar resonances in galactic disks. Here we confirm the efficiency of this mechanism in fully self-consistent, Tree-SPH simulations, as well as high-resolution pure N-body simulations. In all barred cases we clearly identify the effect of spiral-bar resonance overlap by measuring a bimodality in the changes of angular momentum in the disk, $\Delta L$, whose maxima are near the bar's corotation and outer Lindblad resonance. This contrasts with the smooth distribution of $\Delta L$ for a simulation with no stable bar present, where strong radial migration is induced by multiple spirals. The presence of a disk gaseous component appears to increase the rate of angular momentum exchange by about $20 \%$. The efficiency of this mechanism is such that galactic stellar disks can extend to over 10 scale-lengths within $1-3$ Gyr in both Milky Way size and low-mass galaxies (circular velocity $\sim 100 \mathrm{~km} \mathrm{~s}^{-1}$ ). We also show that metallicity gradients can flatten in less than 1 Gyr rendering mixing in barred galaxies an order of magnitude more efficient than previously thought.
\end{abstract}

Key words. galaxies: evolution - galaxies: kinematics and dynamics - galaxies: abundances - galaxies: spiral

\section{Introduction}

In the past few decades, discrepancies in the solar neighborhood age-metallicity relation have implied that effective radial migration (i.e., redistribution of angular momentum) must be taking place in the Milky Way disk (Edvardsson et al. 1993; Haywood 2008; Schönrich \& Binney 2009; see Minchev \& Famaey 2010, for a comprehensive discussion). In parallel, there is now considerable observational evidence that the non-axisymmetry of the Galactic potential can cause significant perturbations in the motion of stars of all ages. Evidence for this comes from, e.g., the moving groups in the solar neighbourhood (Dehnen 1998) containing stars of very different ages (Famaey et al. 2005) or the non-zero value of the $C$ and $K$ Oort constants for red giant stars in the extended local disk (Olling \& Dehnen 2003; Siebert et al. 2010). All of these can be explained by the effect of resonances associated with a central bar (Minchev et al. 2010, 2007) or spiral structure (SS) (Quillen \& Minchev 2005; Quillen et al. 2010).

Until recently it was accepted that efficient radial mixing of stars in galactic disks was caused solely by transient spirals (Sellwood \& Binney 2002, hereafter SB02). However, Quillen et al. (2009) showed that small satellites on radial, in-plane orbits can cause mixing in the outer disk and thus account for the fraction of low-metallicity stars present in the solar neighborhood (Haywood 2008). Moreover, we have recently demonstrated (Minchev \& Famaey 2010, hereafter MF10) that a strong exchange of angular momentum occurs when a stellar disk is perturbed by a central bar and SS simultaneously: our test-particle simulations allowed us to attribute this effect to the overlap or first and second order resonances of each perturber. The presence of multiple patterns in galactic disks has been observed both in external galaxies (e.g., Elmegreen et al. 1992) and N-body simulations (e.g., Rautiainen \& Salo 1999). Given that more than two-thirds of disk galaxies, including our own Milky Way, contain both central bars and SS, it is imperative to establish a strong understanding of the implications of this mechanism. Therefore, in this work we study a range of fully self-consistent, Tree-SPH simulations, as well as high resolution pure N-body simulations, searching for the signature of spiral-bar interaction in the distribution of angular momentum: MF10 predicted that when both bar and spirals are present in the disk, one finds that the changes in angular momentum form a bimodal distribution, with two local maxima close to the corotation and outer Lindblad resonance of the bar, regardless of the pattern speed of the spiral.

\section{Models and results}

While not self-consistent, test-particle simulations allow for a full control over the simulation parameters, such as the amplitudes and pattern speeds of bar and SS, and still provide a good approximation to self-consistent simulations. Employing this method in MF10, we were able to suppress the effect of transient spirals and thus identify the non-linear effect of resonance overlap. We showed that the most important signature of this mechanism was a bimodality in the changes in angular momentum in the disk, $\Delta L$, with maxima near the bar's corotation and its outer Lindblad resonance (OLR) regardless of the SS pattern speed. 
Hereafter, we analyze self-consistent simulations with strong bars and spirals, therefore mixing from both transient spirals (SB02) and resonance overlap (MF10) is expected. However, we hope to be able to identify the latter mechanism by detecting the aforementioned bimodality, given that the predicted distribution of $\Delta L$ generated by transient spirals without a bar is rather smooth and not bimodal (see SB02).

\subsection{Tree-SPH simulation of a giant Sa spiral galaxy}

We test the predictions of MF10 by analyzing fully selfconsistent simulations of isolated disk galaxies from the GalMer database $^{1}$, including a gas component as well as star formation (Di Matteo et al. 2007; Chilingarian et al. 2010).

In Galmer, for each galaxy type, the halo and the optional bulge are modeled as Plummer spheres, with characteristic masses $M_{\mathrm{H}}$ and $M_{\mathrm{B}}$, and characteristic radii $r_{\mathrm{H}}$ and $r_{\mathrm{B}}$, respectively. Their densities are given by:

$$
\rho_{\mathrm{H}}(r)=\left(\frac{3 M_{\mathrm{H}}}{4 \pi r_{\mathrm{H}}^{3}}\right)\left(1+\frac{r^{2}}{r_{\mathrm{H}}^{2}}\right)^{-5 / 2}
$$

and

$$
\rho_{\mathrm{B}}(r)=\left(\frac{3 M_{\mathrm{B}}}{4 \pi r_{\mathrm{B}}^{3}}\right)\left(1+\frac{r^{2}}{r_{\mathrm{B}}^{2}}\right)^{-5 / 2} .
$$

On the other hand, the gaseous and stellar disks follow Miyamoto-Nagai density profiles with masses $M_{\mathrm{g}}$ and $M_{*}$ and vertical and radial scale lengths given, respectively, by $h_{\mathrm{g}}$ and $a_{\mathrm{g}}$, and $h_{*}$ and $a_{*}$ :

$$
\begin{aligned}
\rho_{\mathrm{g}}(R, z)= & \left(\frac{h_{\mathrm{g}}{ }^{2} M_{\mathrm{g}}}{4 \pi}\right) \\
& \times \frac{a_{\mathrm{g}} R^{2}+\left(a_{\mathrm{g}}+3 \sqrt{z^{2}+h_{\mathrm{g}}{ }^{2}}\right)\left(a_{\mathrm{g}}+\sqrt{z^{2}+h_{\mathrm{g}}^{2}}\right)^{2}}{\left[a_{\mathrm{g}}^{2}+\left(a_{\mathrm{g}}+\sqrt{z^{2}+{h_{\mathrm{g}}}^{2}}\right)^{2}\right]^{5 / 2}\left(z^{2}+{h_{\mathrm{g}}}^{2}\right)^{3 / 2}}(3) \\
\rho_{*}(R, z)= & \left(\frac{h_{*}{ }^{2} M_{*}}{4 \pi}\right) \\
& \times \frac{a_{*} R^{2}+\left(a_{*}+3 \sqrt{z^{2}+h_{*}^{2}}\right)\left(a_{*}+\sqrt{z^{2}+h_{*}^{2}}\right)^{2}}{\left[a_{*}^{2}+\left(a_{*}+\sqrt{z^{2}+h_{*}^{2}}\right)^{2}\right]^{5 / 2}\left(z^{2}+h_{*}{ }^{2}\right)^{3 / 2}}(4)
\end{aligned}
$$

\subsubsection{Birth radii of stars}

Here, we first analyze the properties of the isolated giant $\mathrm{Sa}$ (gSa) galaxy model: it has a total mass of $\sim 2.4 \times 10^{11} M_{\odot}$, with $M_{\mathrm{H}}=1.15 \times 10^{11} M_{\odot}, M_{\mathrm{B}}=0.23 \times 10^{11} M_{\odot}, M_{\mathrm{g}}=$ $0.09 \times 10^{11} M_{\odot}$, and $M_{*}=0.92 \times 10^{11} M_{\odot}$. The scale radii are $r_{\mathrm{H}}=10 \mathrm{kpc}, r_{\mathrm{B}}=2 \mathrm{kpc}, h_{\mathrm{g}}=0.2 \mathrm{kpc}, a_{\mathrm{g}}=5 \mathrm{kpc}, h_{*}=0.5 \mathrm{kpc}$, and $a_{*}=4 \mathrm{kpc}$. The initial Toomre parameter of both stars and gas is taken to be $Q=1.2$ as the initial condition of the TreeSPH simulations. Figure 1 shows the properties of this isolated $\mathrm{gSa}$ model as a function of time.

\subsubsection{Identification of the bimodality}

In the first row of Fig. 1, we plot stellar number density contours for five time outputs up to $3 \mathrm{Gyr}$ as indicated in each panel.

\footnotetext{
1 http://galmer.obspm.fr
}

The bottom row of Fig. 1 shows the distribution of birth radii for stars ending up in the annuli indicated by the green lines $(600 \mathrm{pc})$ at $t=2.5 \mathrm{Gyr}$. The black and red histograms show stars on nearly circular orbits (of velocity dispersion smaller than $10 \mathrm{~km} \mathrm{~s}^{-1}$ ) and the total population, respectively. In all cases, a large fraction of stars are found to originate from the bar's corotation (dotted line), as well as an increasingly large fraction from the bar's OLR (solid line) as larger final radii are sampled.

We note that the initially strong SS disappears by the end of the simulation. The expansion of the disk is already an indication of

The second row of Fig. 1 shows contours of the changes in the angular momentum of stars, $\Delta L$, versus the initial angular omentum, $L_{0}$. Both $\Delta L$ and $L$ are divided by the asymptotic

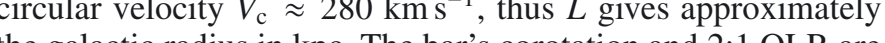
indicated by the dotted and solid lines. A bimodal distribution becomes apparent as soon as the bar and SS form $(t \approx 0.2 \mathrm{Gyr})$. While the peak at $L_{0} \approx 4.5$ is caused by the bar's corotation, the ne at $L_{0} \approx 8.5$ could only be produced by resonance overlap that the effect we see is caused by the spiral-bar interaction. We note also that the mixing timescale here is simply too short to be the effect of transients only.

\subsubsection{Extension of the disk}

The third row of Fig. 1 shows the temporal evolution of the radial density (left) and metallicity (right) profiles for both the stellar gaseous disks. The time steps shown are as in the first row, indicated by solid red, dotted orange, dashed green, dotted-dash e, and solid purple in increasing order.

ng to the vigorous mixing, the disk grows rapidly with sively to larger radii. At the final time (purple lines), the stellar and gaseous disks have extended to about ten and seven scalelengths, respectively, while roughly preserving their exponential profiles. This is accompanied by a strong flattening in the metalicity gradients, where at $t=3 \mathrm{Gyr}$ one can clearly see a reversal $(\sim 30 \mathrm{kpc})$. The stellar metarion the initial ones at $8 \mathrm{kpc}$, suggesting that stars in the outskirts of the disk originate from regions near the bar's 2:1 OLR, where he changes in $\Delta L$ are most prominent.

\subsection{Tree-SPH simulations of other galaxy types}

To assess how our results depend on the peculiar galaxy type we considered, we plot in Fig. 2 the changes in angular momentum for the stellar disk components of all GalMer isolated giant galaxy models available (Chilingarian et al. 2010, Table 1). Contour levels are the same for all plots and the time is $t=1 \mathrm{Gyr}$. All apart from the gSd (bulgeless) model develop long-lived, central bars at the beginning of the simulation and consequently show a bimodality in $\Delta L$. When we compare the gS0 and gSa models, which have identical initial conditions except that gS0 is gas-free, we find that $\Delta L$ increases by $\sim 20 \%$ when gaseous disk component is introduced. The stability of the gas, however, does not appear to play a major role. Only a small difference (see Fig. 2 in MF10). At all later times this clear bimodality re- 
I. Minchev et al.: Mixing in galactic disks caused by multiple patterns
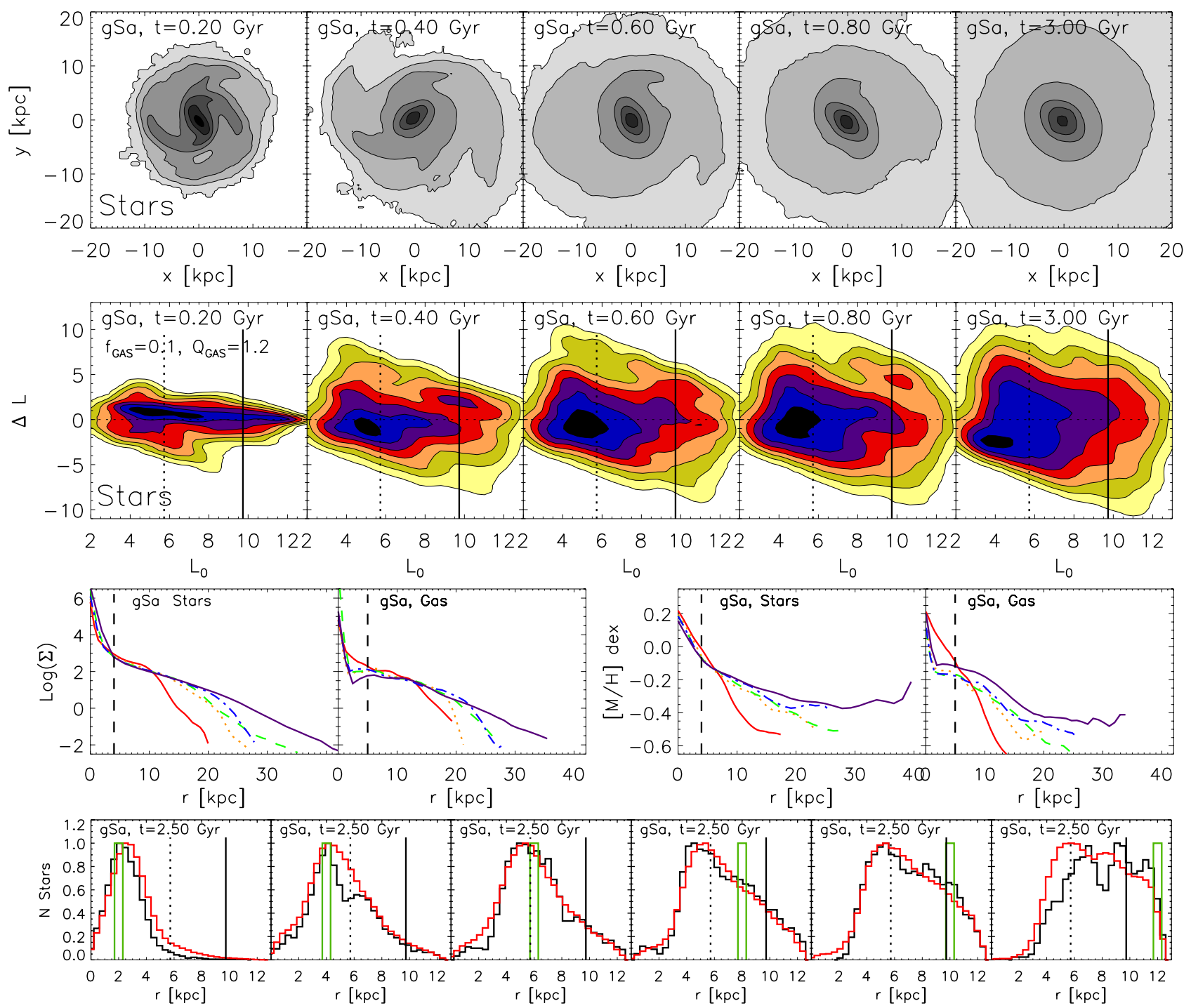

Fig. 1. First row: stellar disk number density contours of the isolated giant Sa galaxy simulation in GalMer for 5 time outputs as indicated in each panel. Second row: changes in angular momentum, $\Delta L$ as a function of the initial angular momentum, $L$. Both $\Delta L$ and $L$ are divided by the asymptotic circular velocity $V_{\mathrm{c}}$, thus $L$ gives approximately the galactic radius in kpc. The locations of the bar's corotation and 2:1 OLR are indicated by the dotted and solid lines, respectively. A bimodal distribution indicating the work of resonance overlap of bar and spirals is clearly seen in each panel. Third row: the evolution of the radial profiles (left) and metallicities (right) for the stellar and gaseous disks. The initial stellar and gaseous disk scale-lengths are indicated by the dashed lines. The time steps shown are as in the first row, indicated by solid red, dotted orange, dashed green, dotted-dash blue, and solid purple lines, respectively. Fourth row: distribution of birth radii for stars ending up in the annuli indicated by the green lines $(600 \mathrm{pc})$ at $t=2.5 \mathrm{Gyr}$.

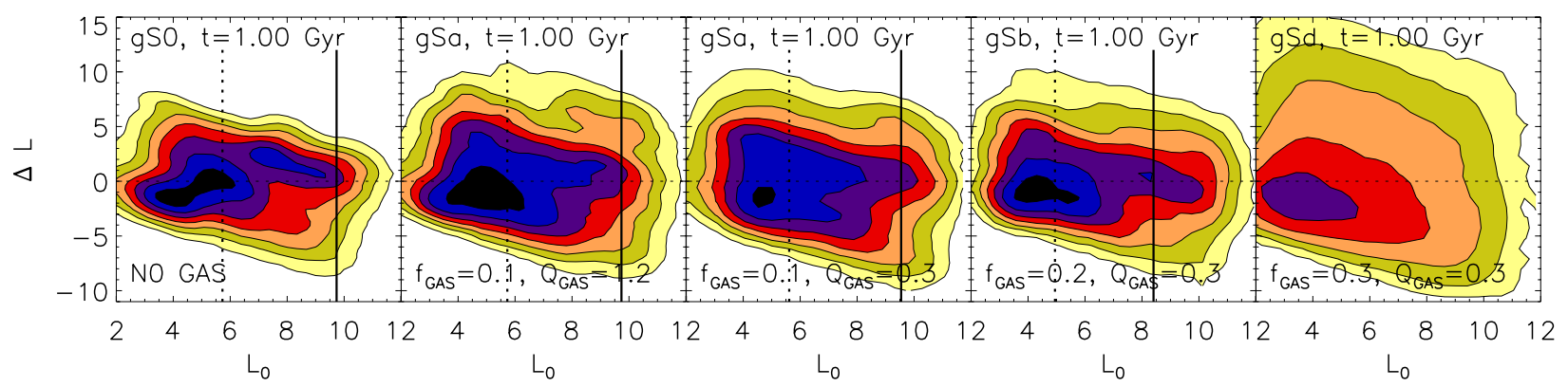

Fig. 2. Same as the second row of Fig. 1 but for all isolated giant GalMer galaxies. Contour levels are the same for all plots and the time is $t=1 \mathrm{Gyr}$. The locations of the bar's corotation and OLR are indicated by the dotted and solid lines, respectively. The rightmost panel shows the only disk lacking a central bar. In this case, resonance overlap from strong multiple spirals gives rise to a smooth $\Delta L$ distribution.

occurs when the gas is made more unstable when $Q_{\text {gas }}$ is lowered from 1.2 to 0.3 (second and third panels), where $Q_{\text {gas }}$ is the
Toomre instability parameter for the gas. The weaker bar in the gSb model (fourth panel) results in reduced mixing as we 


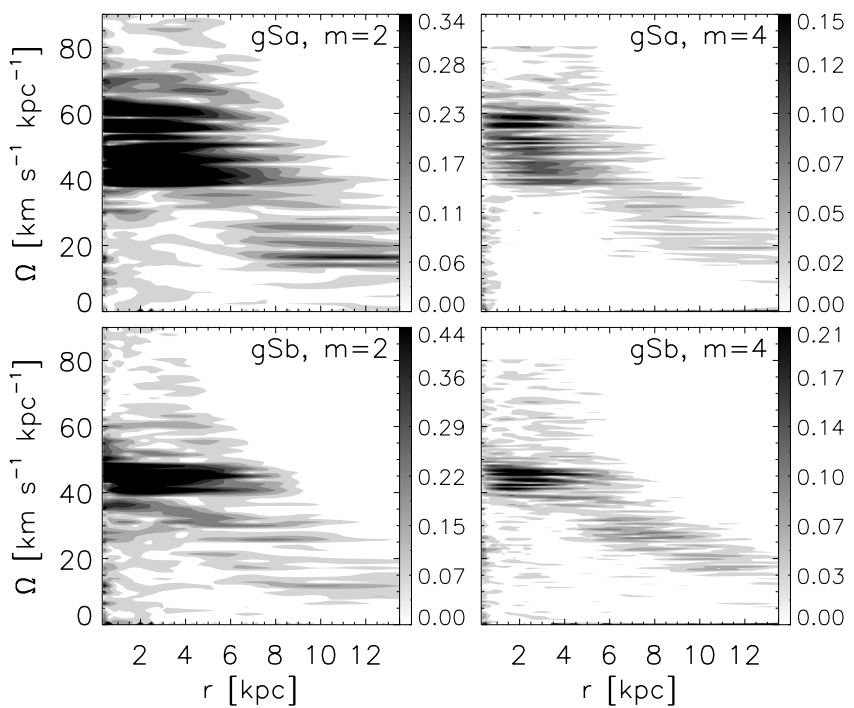

Fig. 3. Power spectrum displaying the pattern speed of the $m=2$ and $m=4$ Fourier components, during the entire simulation, of the gSa (top) and gSb (bottom) models. Contour levels are indicated for each panel. Note that the gSa bar evolves (slows down and extends) much more and its two-armed SS is twice as strong as for the gSb model (top left). Despite the slightly stronger gSb bar, the effect on $\Delta L$ in the gSa's outer disk is much more prominent (Fig. 2) because of the combined effect of the bar and stronger spirals.

predicted in MF10. Unlike all other models, the gSd simulation (rightmost panel) does not form a stable bar. The strong mixing observed here results from the large number of multiple spirals (Elmegreen et al. 1992; Rix \& Rieke 1993; Minchev \& Quillen 2006) propagating simultaneously through the disk during the first 500 Myr. Due to the various SS pattern speeds, there are no radii at which the effect of resonance overlap differs distinctly from the rest of the disk (such as the bar's corotation and OLR). Consequently, we observe a smooth distribution of $\Delta L$ in contrast to the case of bar $+\mathrm{SS}$.

\subsection{Pattern speeds}

Figure 3 shows a power spectrum displaying the pattern speeds of the $m=2$ and $m=4$ (two-armed and four-armed structure) Fourier components, over the whole simulation, of the gSa (top) and gSb (bottom) models. Contour levels are indicated for each panel in units of $Q_{\mathrm{T}}$, the ratio of the maximum tangential force to the azimuthally averaged radial force at a given radius. For both simulations, the $\mathrm{SS}(r \gtrsim 5 \mathrm{kpc})$ spans a range of pattern speeds, always slower than the bar. As expected from the results of MF10, the SS pattern speed has little influence on the location of the maxima in the bimodal distribution of $\Delta L$. Note that the gSa bar evolves (slows down and extends over time) much more, and that its two-armed SS is twice stronger compared to the gSb model (top left). Despite the slightly stronger $\mathrm{gSb}$ bar, the effect on $\Delta L$ for the $\mathrm{gSa}$ is much more prominent in the outer disk (Fig. 2) because of the combined effect of the bar and stronger spirals. Strong spirals are thus needed for this migration mechanism to be efficient. However, the relation between the strength of the perturbers and the angular momentum redistribution is nonlinear as previously shown by MF10.

\subsection{High resolution $\mathrm{N}$-body simulations}

To determine whether the strong mixing we described above is caused by a deficiency in the resolution of the GalMer

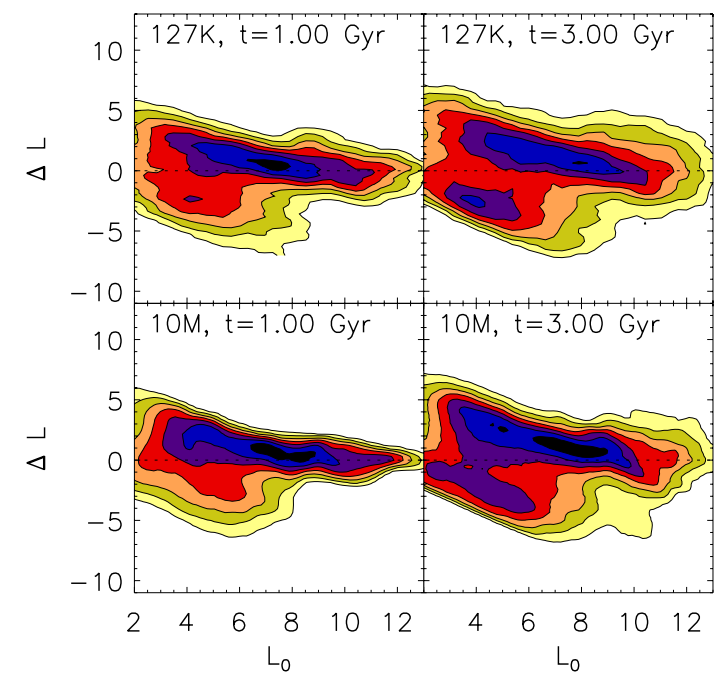

Fig. 4. Contrasting the effect on $\Delta L$ for a low-resolution $\left(1.27 \times 10^{5}\right)$ and a high-resolution $10^{7}$ pure $N$-body simulations.

simulations, we ran pure $N$-body collisionless simulations with $10^{7}$ and $1.27 \times 10^{5}$ particles in the disk. Full description and details can be found in Wozniak \& Michel-Dansac (2009). With a scale-length of $3.5 \mathrm{kpc}$, the initial conditions of the disk are comparable to those of the gSb GalMer model (Fig. 2) but lack a gas component. These simulations have no halo, which causes $\mathrm{a} \sim 30 \%$ drop in the RC at $\sim 10 \mathrm{kpc}$.

Figure 4 shows the changes in angular momentum at $t=1$ and $3 \mathrm{Gyr}$ for $1.27 \times 10^{5}$ (top) and $10^{7}$ (bottom) disk particles. At the beginning of the simulations, the effect is stronger for the low-resolution case due to the faster bar formation. However, at $t \approx 2$ Gyr both the high- and low-resolution runs yield a similar result. This suggests that the magnitude of the radial migration induced by the bar-spiral interaction and the timescale over which it is effective are not strongly dependent on the numerical resolution of the simulations. We conclude that the properties of the mixing, i.e., its magnitude and timescale, that we infer from the GalMer simulations are only weakly affected by the insufficient resolution.

\subsection{Migration in low-mass galaxies}

We now investigate whether the resonance overlap mechanism is also efficient in low-mass galaxies. Gogarten et al. (2010) showed that although transient spirals can explain extended disks for MW-type galaxies, this mixing mechanism is inefficient for galaxies with RCs of $V_{\mathrm{c}} \sim 100 \mathrm{~km} \mathrm{~s}^{-1}$, such as NGC 300 and M 33. Nevertheless, both NGC 300 and M 33 are observed to have extended radial profiles of up to ten scale-lengths. We now consider the barred, dwarf $\mathrm{Sa}(\mathrm{dSa})$ simulation in GalMer (RC of $100 \mathrm{~km} \mathrm{~s}^{-1}$ and initial scale-length of $1.3 \mathrm{kpc}$ ) to see how its density distribution and metallicity gradient evolve with time. In Fig. 5 we plot the time development of the stellar disk density and metallicity profiles, for the same time steps as in Fig. 1. We can clearly see that the stellar disk extends to more than ten scale-lengths in $\sim 3 \mathrm{Gyr}$, while preserving its exponential mass density profile. At the same time, the metallicity gradient becomes flat in less than 1 Gyr. Both the predicted extent of the stellar disk and the flattening of the metallicity profile are consistent with what has been recently reported for nearby lowmass galaxies (Bland-Hawthorn et al. 2005; Vlajić et al. 2009). 

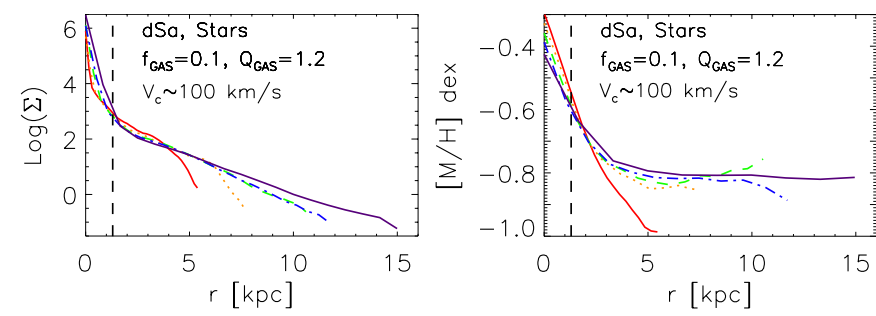

Fig. 5. The time evolution of the stellar disk density (left) and metallicity (right) profiles for the dwarf Sa isolated GalMer model. Time outputs and colors are the same as the third row of Fig. 1. This is a low-mass galaxy with a rotation curve of $\sim 100 \mathrm{~km} \mathrm{~s}^{-1}$ similar to NGC 300 and M 33. The initial disk scale-length is indicated by the dashed line. The disk extends to over ten scale-lengths in less than $3 \mathrm{Gyr}$.

\section{Conclusions}

We have examined the redistribution of angular momentum in galactic disks by means of Tree-SPH and high-resolution pure $\mathrm{N}$-body simulations. We have found that resonance overlap of multiple patterns (such as bar $+\mathrm{SS}$ or SS $+\mathrm{SS}$ ) induces strong exchange of angular momentum throughout the disks in agreement with the predictions of MF10. Since in the self-consistent simulations analyzed in this work spirals may be transient, we should also expect a contribution from the SB02 radial migration mechanism. However, for the short timescales considered here $(<1 \mathrm{Gyr})$ transients would simply have a very small effect (SB02). The resonance overlap mechanism is clearly identified by a bimodality in the changes in angular momentum, $\Delta L$, caused by the bar's corotation and 2:1 OLR (Figs. 1, 2, and 4). We have contrasted this to a simulation lacking a stable central bar (Fig. 2, rightmost panel), where the $\Delta L$ distribution is smooth. The effect is especially strong when a gaseous component is present, as a result of the exchange of $L$ between gas and stars coming from the gravity torques, the phase shift between the two components, and the gas dissipation (Bournaud \& Combes 2002). Depending on the amount of gas and the strength of the bars and spirals, the metallicity gradients can flatten in less than 1 Gyr (Fig. 1). This is in drastic contrast to the current understanding that galactic disks need a Hubble time for sufficient mixing (SB02, Roškar et al. 2008).

How can we tie our results to galactic disk evolution? Bournaud \& Combes (2002) followed the detailed processes of bar formation, bar destruction, and bar re-formation, while varying the disk to bulge ratio. These authors identified three bar formation episodes in a Hubble time. In this model, we can regard a given GalMer isolated galaxy simulation as one such episode of a gas accretion event during a galaxy lifetime. In this scenario, the rapid flattening of the metallicity gradients expected from the vigorous migration (Fig. 1) would be followed by a gas enrichment at each bar re-formation event resulting in the rebuilding of the gradient. The presence or not of a metallicity gradient, or its intensity, would then be an indicator of the bar/accretion phase of the galaxy.

The mechanism described in this study works even in lowmass galaxies (Fig. 5) and can thus for the first time provide an explanation for the extended disk profiles observed in galaxies with $V_{\mathrm{c}} \sim 100 \mathrm{~km} \mathrm{~s}^{-1}$ (Bland-Hawthorn et al. 2005; Minchev et al., in prep.).

Acknowledgements. We would like to thank Alice Quillen, Cristina Chiappini, Ken Freeman and Joss Bland-Hawthorn for their valuable comments. Support for this work was provided by ANR-CNRS, and the AvH foundation.

\section{References}

Bland-Hawthorn, J., Vlajić, M., Freeman, K. C., \& Draine, B. T. 2005, ApJ, 629, 239

Bournaud, F., \& Combes, F. 2002, A\&A, 392, 83

Chilingarian, I., Di Matteo, P., Combes, F., Melchior, A., \& Semelin, B. 2010, A\&A, 518, A61

Dehnen, W. 1998, AJ, 115, 2384

Di Matteo, P., Combes, F., Melchior, A., \& Semelin, B. 2007, A\&A, 468, 61

Edvardsson, B. 1993, A\&A, 275, 101

Elmegreen, B. G., Elmegreen, D. M., \& Montenegro, L. 1992, ApJS, 79, 37

Famaey, B., Jorissen, A., Luri, X., et al. 2005, A\&A, 430, 165

Gogarten, S. M. 2010, ApJ, 712, 858

Haywood, M. 2008, MNRAS, 388, 1175

Minchev, I., \& Quillen, A. C. 2006, MNRAS, 368, 623

Minchev, I., \& Famaey, B. 2010, ApJ, 722, 112 (MF10)

Minchev, I., Nordhaus, J., \& Quillen, A. C. 2007, ApJ, 664, L31

Minchev, I., Boily, C., Siebert, A., \& Bienayme, O. 2010, MNRAS, 407, 2122

Olling, R., \& Dehnen, W. 2003, ApJ, 599, 275

Quillen, A. C., \& Minchev, I. 2005, AJ, 130, 576

Quillen, A. C., Minchev, I., Bland-Hawthorn, J., \& Haywood, M. 2009, MNRAS, 397, 1599

Quillen, A. C., Dougherty, J., Bagley, M. B., Minchev, I., \& Comparetta, J. 2010, MNRAS, submitted [arXiv: 1010.5745]

Rautiainen, P., \& Salo, H. 1999, A\&A, 348, 737

Rix, H., \& Rieke, M. J. 1993, ApJ, 418, 123

Roškar, R., Debattista, V. P., Quinn, T. R., Stinson, G. S., \& Wadsley, J. 2008, ApJ, 684, L79

Schönrich, R., \& Binney, J. 2009, MNRAS, 396, 203

Sellwood, J. A., \& Binney, J. J. 2002, MNRAS, 336, 785

Siebert, A., Famaey, B., Minchev, I., et al. 2011, MNRAS, accepted [arXiv: 1011.4092]

Vlajić, M., Bland-Hawthorn, J., \& Freeman, K. C. 2009, ApJ, 697, 361

Wozniak, H., \& Michel-Dansac, L. 2009, A\&A, 494, 11 